ص ص ص
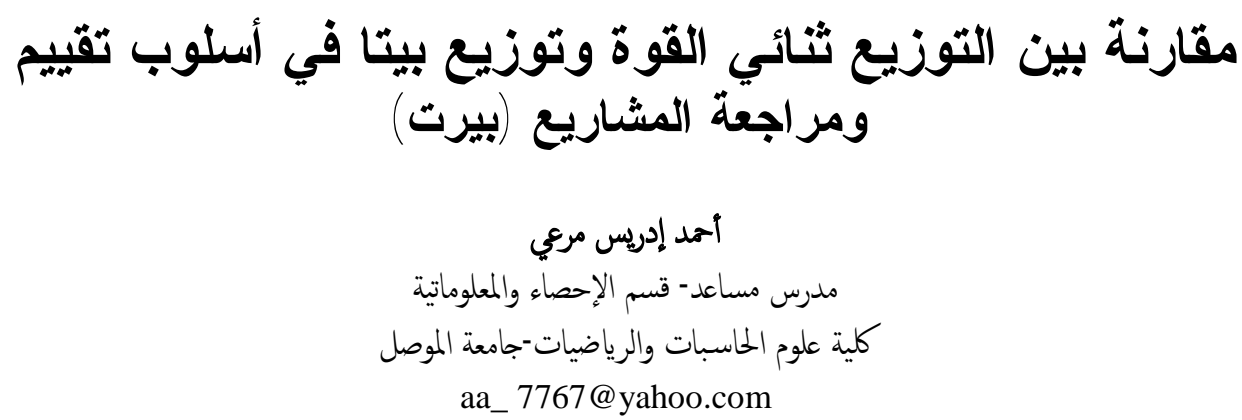

\title{
Comparison between Two - sided Power Distribution and Beta Distribution in Program Evaluation and Review Technique (PERT)
}

\author{
Ahmed I. Maree \\ Assistant Lecturer \\ Department of Statistics and Informatic \\ Mosul University
}

\begin{abstract}
The planning process is considered to be one of the most important tasks in our practical life. The project is divided into a number of activities according to the plan. Each activity represents functional process which requires time in order to be carried out. This period of time is considered either limited or probable. The aim of research after studying the probable time of the activities is to find out the suitable distribution for calculating the range of an appropriate of the three uncertain times which include: optimistic, pessimistic

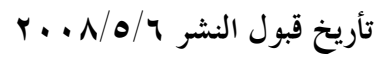

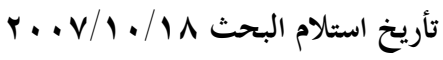


and moderate time. As for Beta distribution It was previously considered a four fold parameter for adequate distribution which was used to estimate the average of the activity of triple times. In this research many exceptional problems have been put aside concerning Beta distribution usages one of them is giving four weights to moderate time compared with one Wight which is given to the optimistic time and another is given to pessimistic time. We have also adapted that method on the present practical \& we have got an outcome which shows suitability of Two - Sided Power Distribution for time and activates.

المقدمة

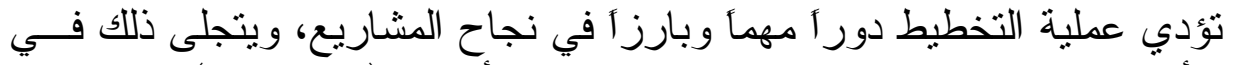

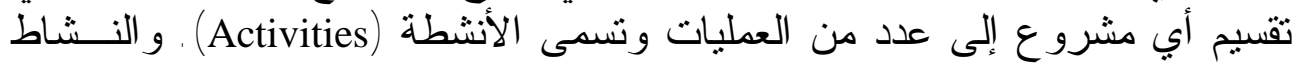

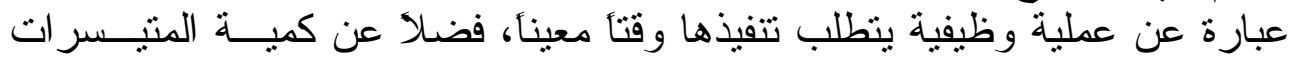

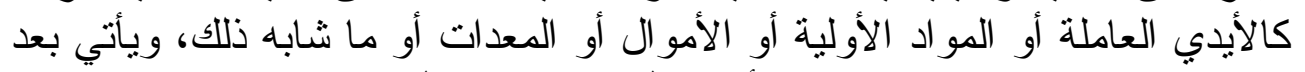

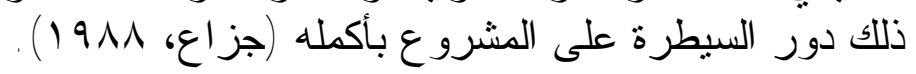

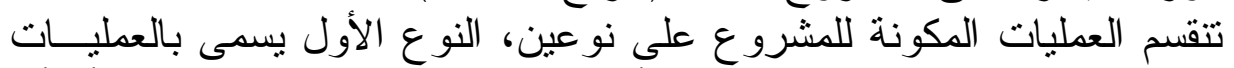

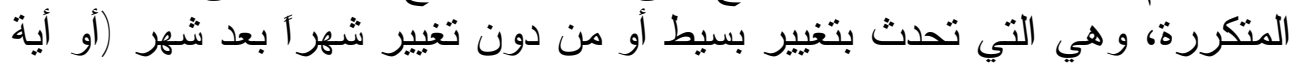

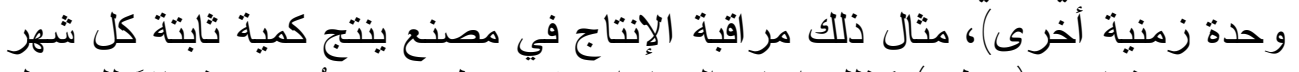

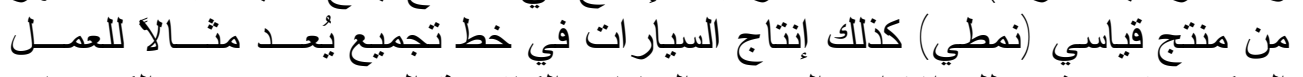

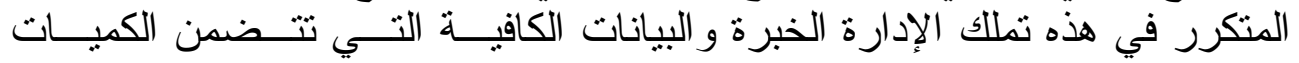

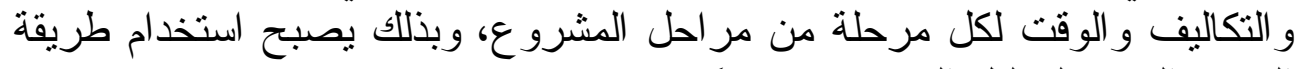
المسار الحرج لتحليل المشاريع مناسباً.

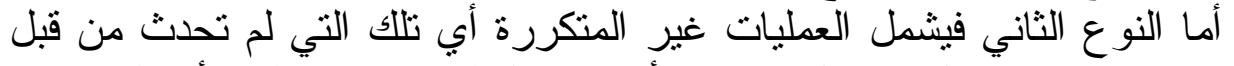

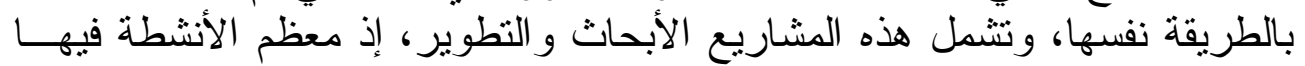

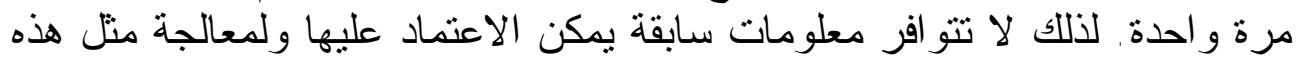

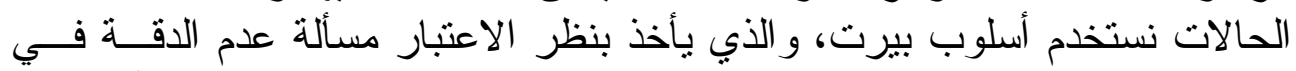

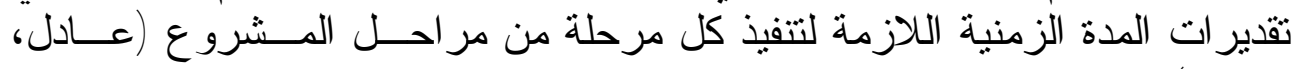

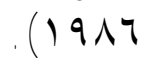

ومن الجدير بالذكر أن أسلوب بيرت يأخذ بنظر الاعتبار الاحتمالات المتعـدـدة الإنة

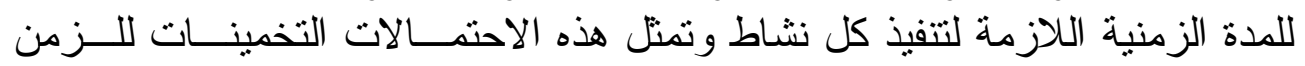

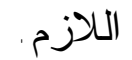

r أسلوب تقييم ومراجعة المشاريع ( بيرت PERT)

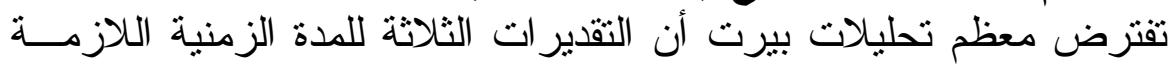

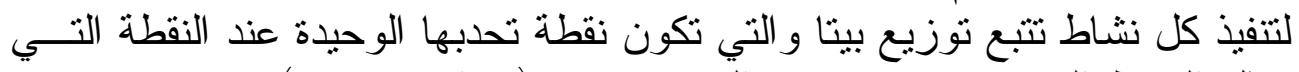
تمنتل الوسط الحسابي ونهايته عند التقديرين a,b (جزاع،

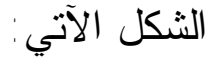




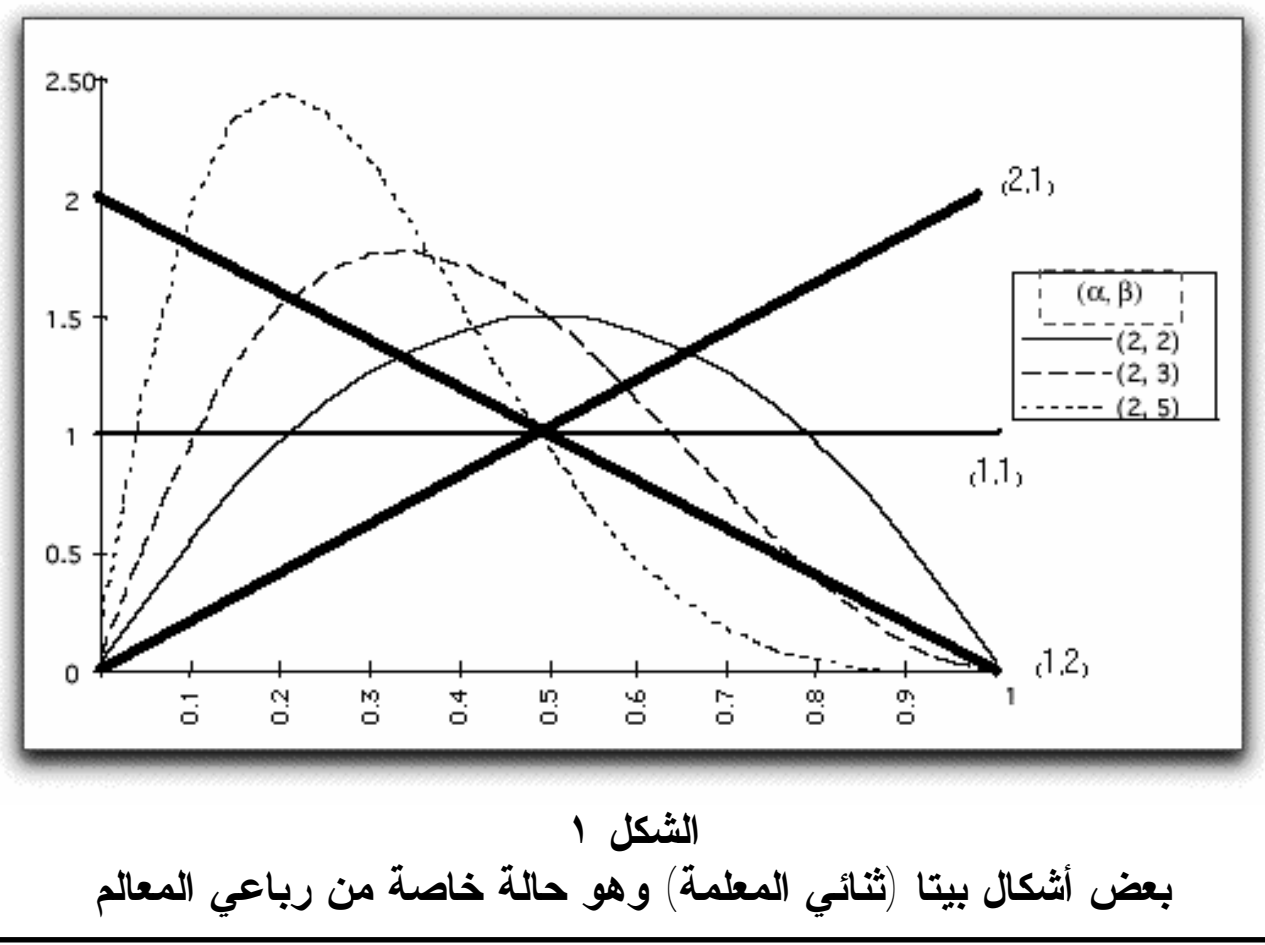

إن توزيع بيتا المستخدم في أسلوب بيرت بملك أربع معالم، ودالـــة الكثافـــة الاحتمالية له هي (Van Dorp, 2002): $f(t ; a, b, \alpha, \beta)=\frac{\Gamma(\alpha+\beta)}{\Gamma(\alpha) \Gamma(\beta)} \frac{(t-\alpha)^{\alpha-1}(b-t)^{\beta-1}}{(b-a)^{\alpha+\beta+1}}$ حيث إن 0 >a,b, 0 و هي معالم التوزيع

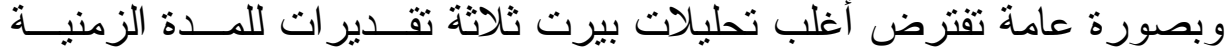

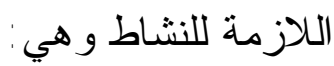

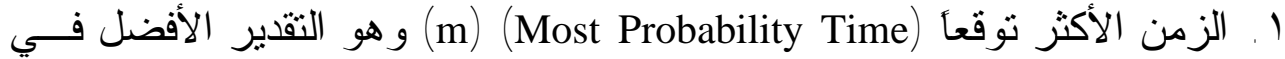
مدة زمنية مدكنة لانجاز النشاط في ظل الظمان الظروف الطبيعية.

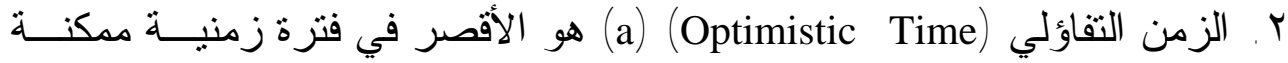

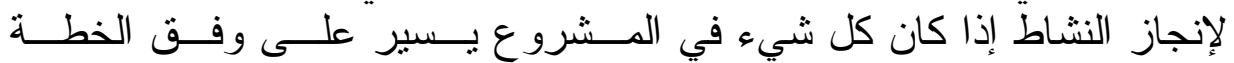

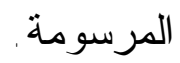

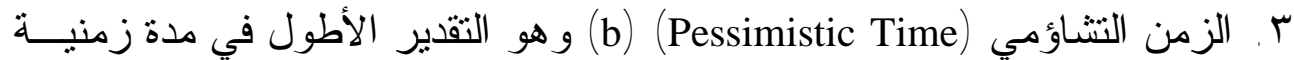

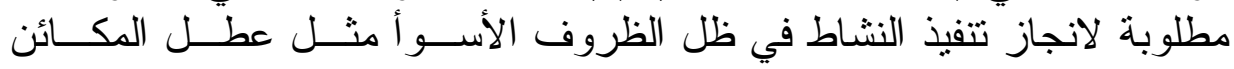

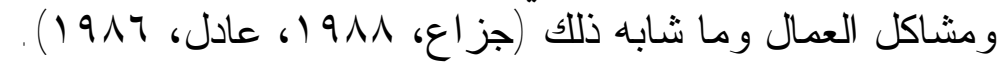

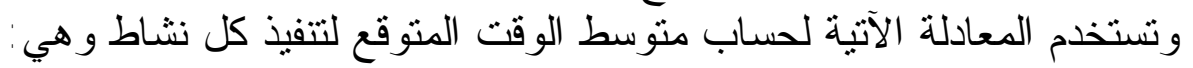




$$
t e=\frac{a+4 m+b}{6}
$$

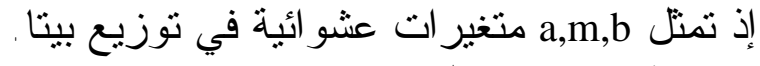

$$
\begin{aligned}
& \text { وصيغة الانحر اف المعياري هي : }
\end{aligned}
$$

$$
\sigma=\frac{(b-a)}{6}
$$

إلا أن الصيغتين السابقتين لم يتم إيجادهما بالاشتقاقات المألوفــــ Bradley،

ويهدف البحث إلى دراسة مقارنة بين الفرضية القائلة بتوزيــع بيتـــا لأوقـات

\section{الأنشطة مع التوزيع ثنائي القوة.}

ويستخدم هذا الأسلوب لتحديد التئ التمال إنجاز المشروع طو ال مدة معينة يرمز لها بالرمز (ST)، و ولى هذا الأساس فإنه:

$$
\operatorname{Pr}(t e \leq S T)=\operatorname{Pr}\left(\frac{t e-\mu}{\sqrt{V(t e)}} \leq \frac{S T-\mu}{\sqrt{V(t e)}}\right)
$$

$$
=\operatorname{Pr}\left(Z \leq D_{i}\right)
$$

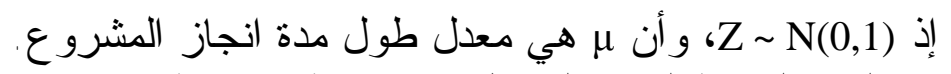

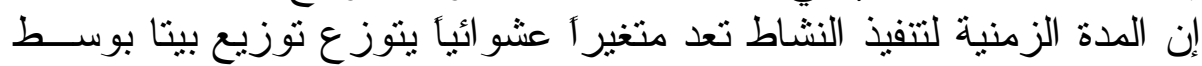

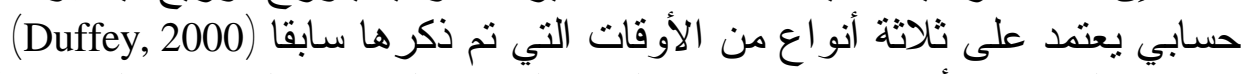

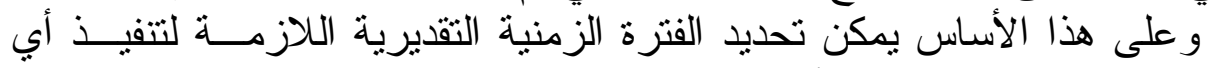
نشاط معتمدين على الصيغة الآتية:

$$
t e=\frac{[(a+b) / 2]+2 m}{3}=\frac{a+4 m+b}{6}
$$

وحسب النظرية القائلة بأن الأوقات الثلاثة تتوزع على وفق توزيع بيتا يـتم حساب التباين على وفق الصيغة الآتية:

$$
\sigma_{i j}^{2}=\left(\frac{b-a}{6}\right)^{2}
$$

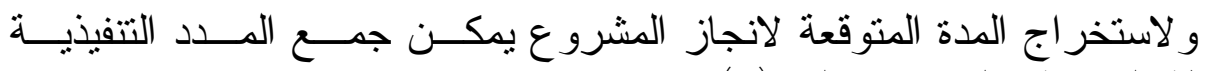

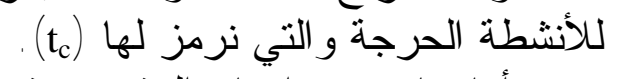

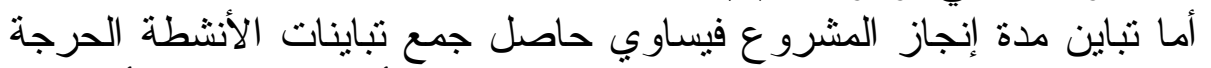
التي نرمز لها (c.p.)

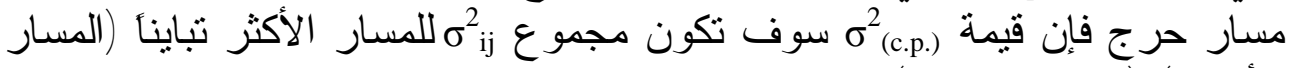




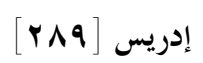

$$
\sigma_{(c . p .)}^{2}=\sum_{i, j \in c . p .} \sigma_{i j}^{2}
$$

\section{ا ـ التوزيع ثنائي القوة}

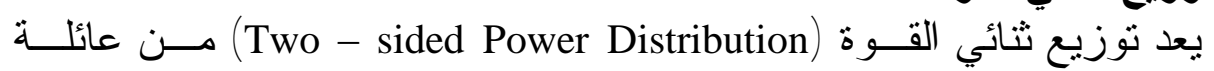
التوزيعات المستمرة المهمة و التي تستخدم بكثرة في المجال الهندسي و المالي، وقد

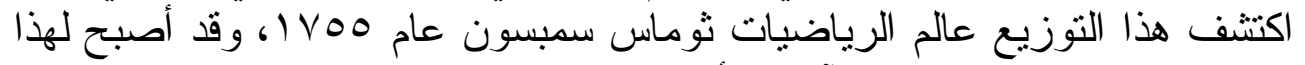

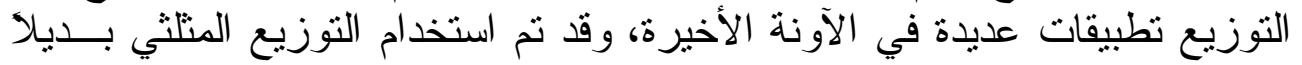

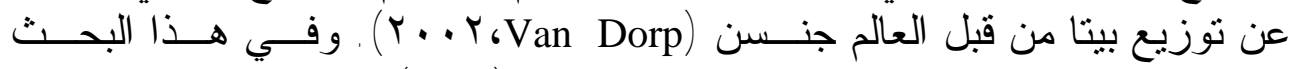

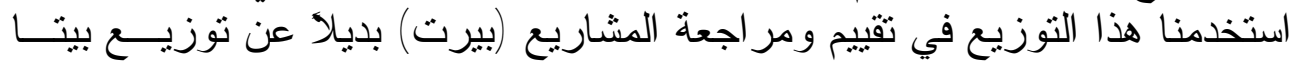

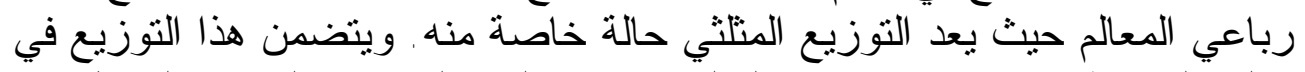

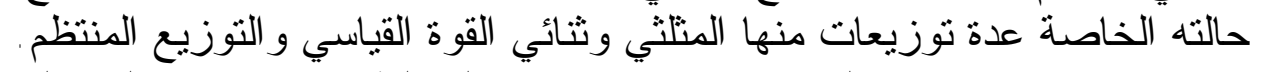

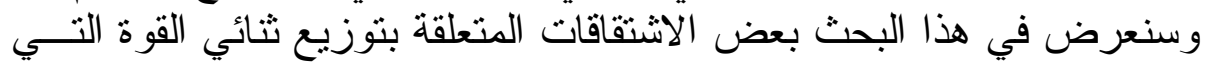

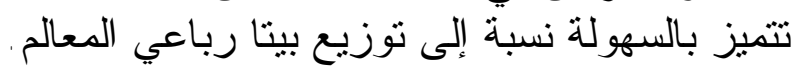
فإذا فرضنا x متغير آ عشو ائياً بدالة كثافة احتمالية معطاة بالثكل الآتي:

$$
f(x \mid a, m, b, n)=\left\{\begin{array}{lll}
\frac{n}{(b-a)}\left(\frac{x-a}{m-a}\right)^{n-1} & \ldots & a \leq x \leq m \\
\frac{n}{(b-a)}\left(\frac{b-x}{m-a}\right)^{n-1} & \ldots & m \leq x \leq b
\end{array}\right.
$$

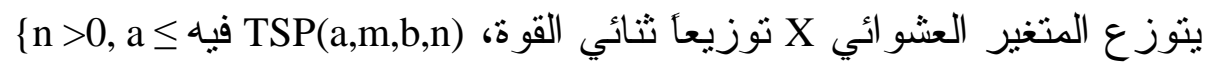

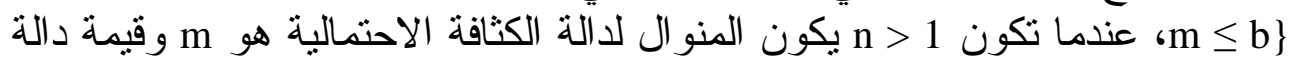

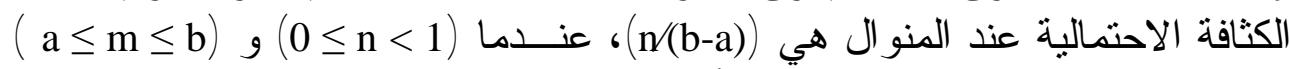

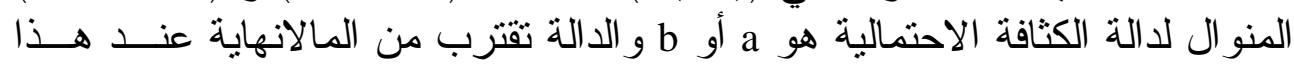

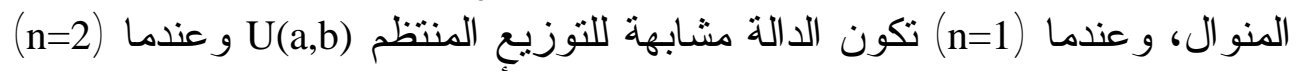

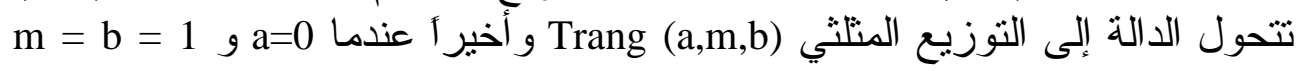

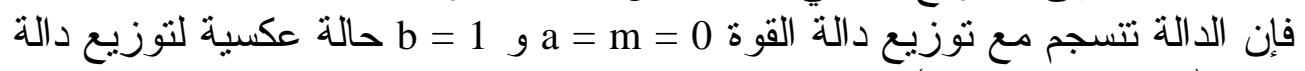
القوة (Van Dorp, 2002). 


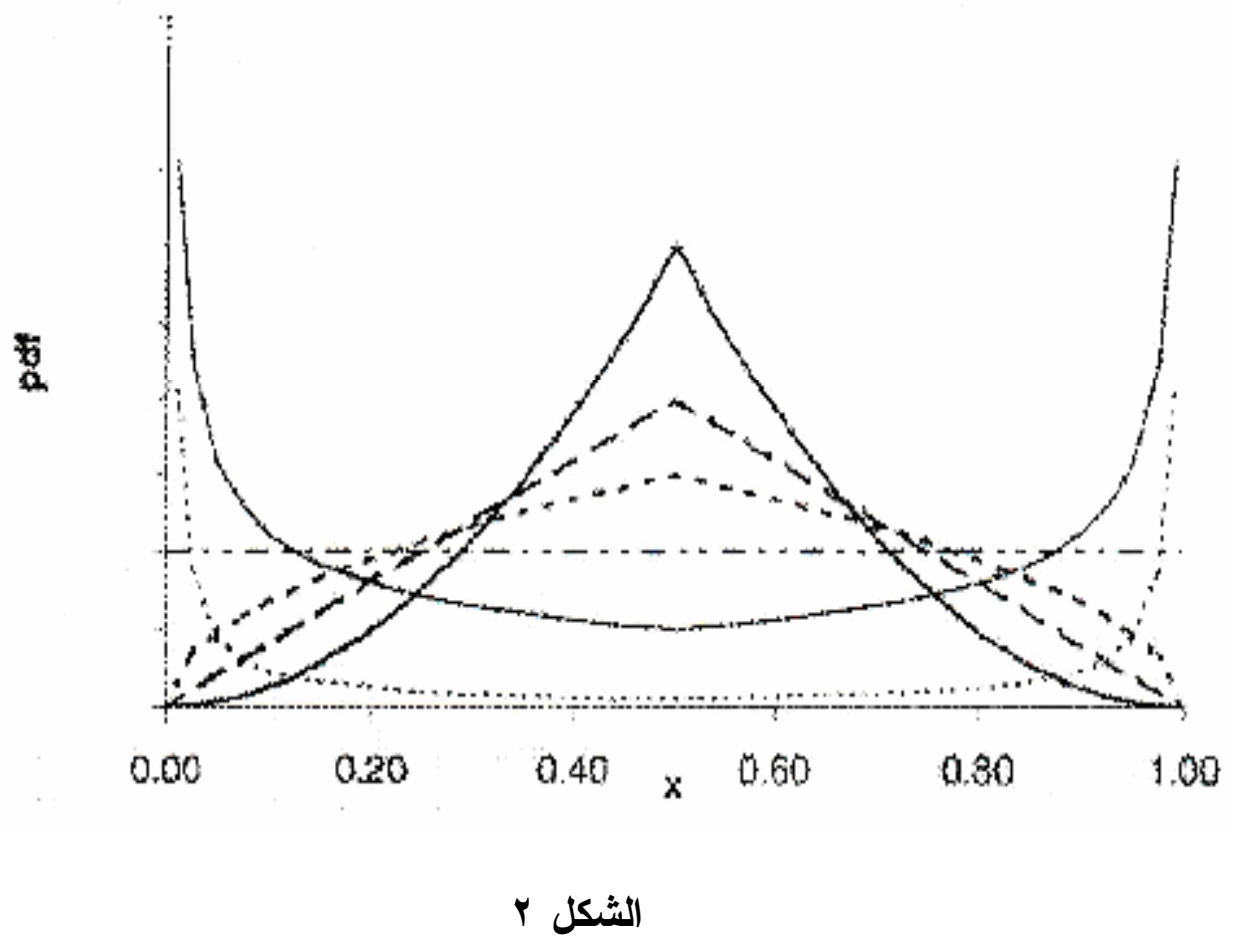

يعرض أمثلة لبعض أثكال التوزيع إذ إنه عندما 0.5 = يتضمن التوزيعات

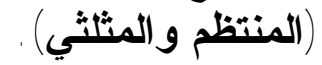

$$
\begin{aligned}
& \mathrm{m}=0.5: \cdots, \mathrm{n}=0.05:-, \mathrm{n}=0.5: \cdots-\cdot \cdot \mathrm{n}=1: \cdots, \\
& \mathrm{n}=1.5:-\longrightarrow, \mathrm{n}=2 \text { : }
\end{aligned}
$$




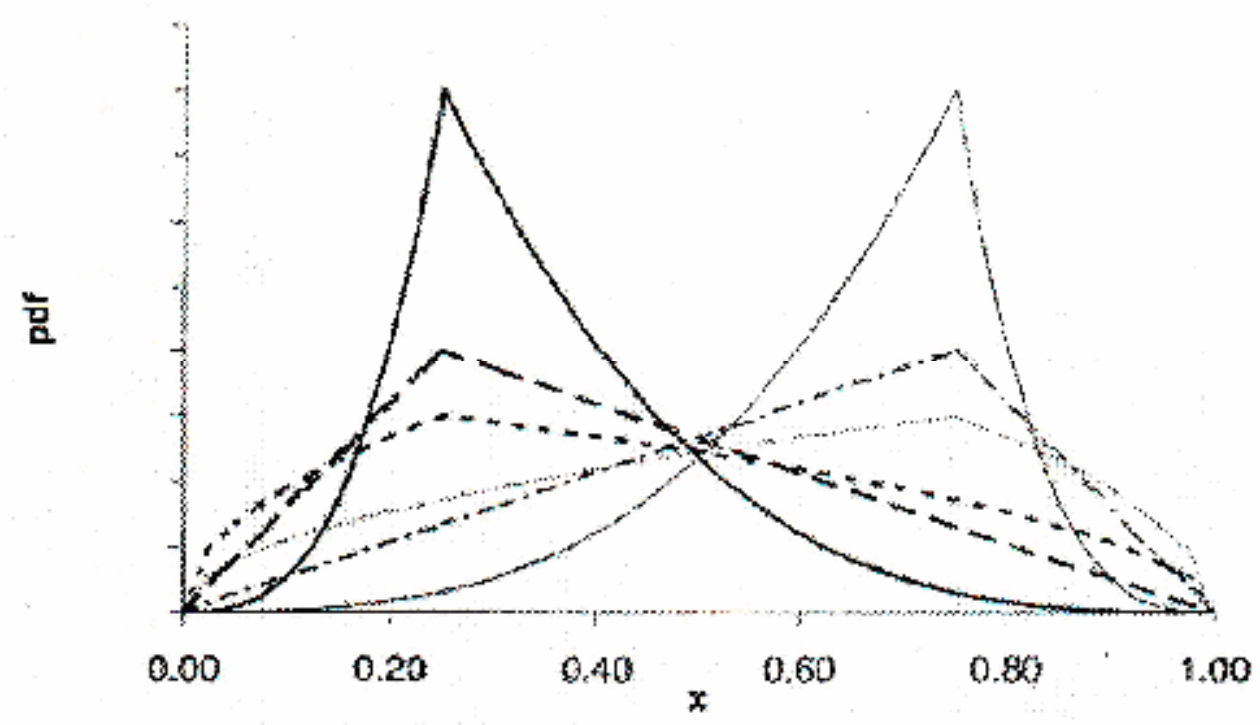

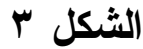

يحتوي على أمثلة ملتوية لليمين و اليسار لتوزيعات (TSP(0,m,1,n)، ويضمنه

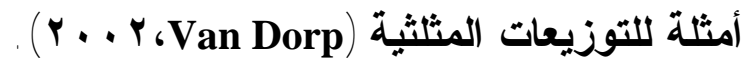

$\mathrm{m}=0.25, \mathrm{n}=1.5:---; \mathrm{m}=0.25, \mathrm{n}=2: \square ; \mathrm{m}=0.25, \mathrm{n}=4: \cdots \cdot \cdots$

$\mathrm{m}=0.75, \mathrm{n}=1.5: \cdots-\cdot ; \mathrm{m}=0.75, \mathrm{n}=4$ :

r - 1 التوقع والتباين للتوزيع ثنائي القوة

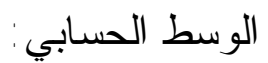

$E(t)=\frac{a+(n-1) m+b}{n+1}$

التباين:

$V(t)=\frac{n(b-a)^{2}-[2(n-1)(m-a)(b-m)]}{(n+2)(n+1)^{2}}$ 


$$
\begin{aligned}
& r . s .=a+(m-a) \times \sqrt[n]{\frac{(b-a)}{(m-a)} U} \text { for } U \leq \frac{(m-a)}{(b-a)} \\
& \text { r.s. }=b-(b-m) \times \sqrt[n]{\frac{(b-a)(1-U)}{(b-m)}} \text { for } U \geq \frac{(m-a)}{(b-a)}
\end{aligned}
$$

$$
\begin{aligned}
& \text { إذ إن: U Uniform } \\
& \text { تعني متغير عشو ائي المولَّد }
\end{aligned}
$$

\section{r r ب دالة التوزيع (الدالة التر اكمية)}

$$
F(x)=\left\{\begin{array}{cc}
0 & \text { for } x<a \\
\frac{m-a}{b-a}\left(\frac{x-a}{m-a}\right)^{n} & \text { for } a \leq x \leq m \\
1-\left(\frac{b-m}{b-a}\right)\left(\frac{b-x}{b-m}\right)^{n} & \text { for } m \leq x<b \\
1 & \text { for } x \geq b
\end{array}\right.
$$

أما بالنسبة لمعالم التوزيع فقد تم تقدير ها بطريقة العـزوم وطريقــــة الإمكـــان

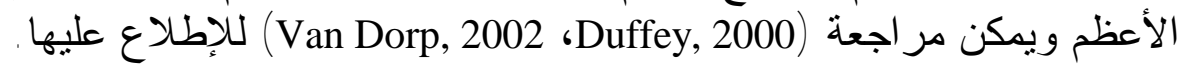

\section{r - ـ مقارنة معالم التوزيع ثنائي القوة مع معالم توزيع بيتا}

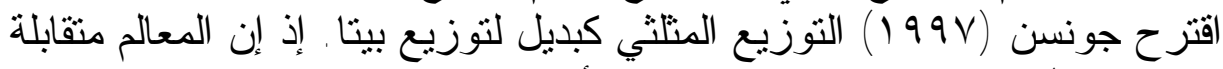

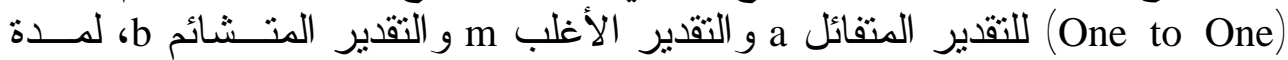

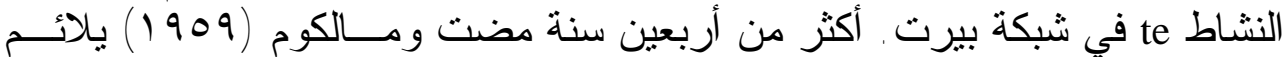

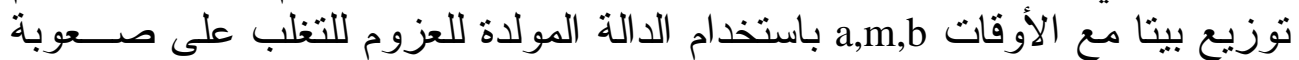
تقدير معالم بيتا و الذي يكون وسطه وتباينه بالثكل الآتي (Duffey, 2000):

$$
\begin{aligned}
& E(t)=\frac{a+4 m+b}{6} \\
& V(t)=\frac{1}{36}(b-a)^{2}
\end{aligned}
$$




\section{[إدريس [ra [ra}

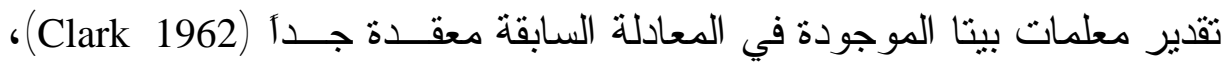

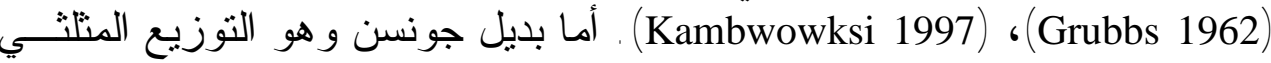
فإن وسطه وتباينه يعطى بالثكل الآتي:

$$
\begin{aligned}
& E(X)=\frac{a+m+b}{3} \\
& V(X)=\frac{1}{18}\left(a^{2}+b^{2}+m^{2}-a b-a m-b m\right) \\
& \frac{3}{72}(b-a)^{2} \leq V(X) \leq \frac{1}{18}(b-a)^{2}
\end{aligned}
$$

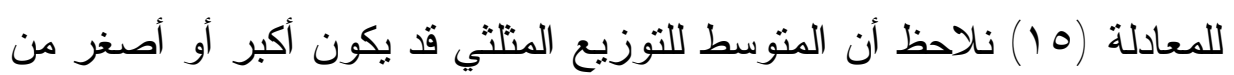

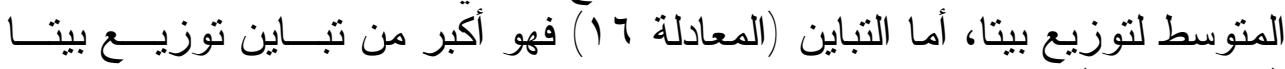

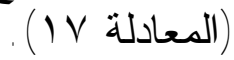
وبدلا من التوزيع المثلثي يمكن استخدام توزيع TSP(a,m,b,n) الأكثر عمومية

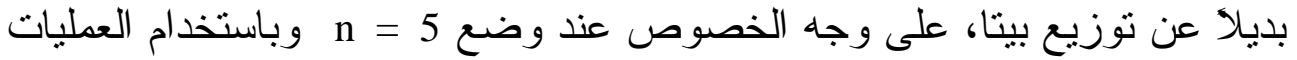

$$
\begin{aligned}
& E(X)=\frac{a+4 m+b}{6} \\
& \frac{1}{84}(b-a)^{2} \leq V(X) \leq \frac{5}{252}(b-a)^{2}
\end{aligned}
$$

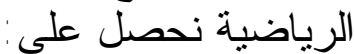

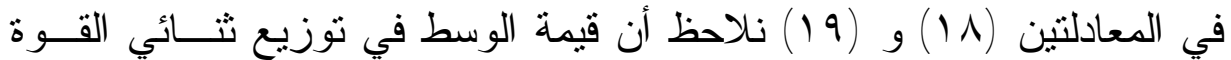

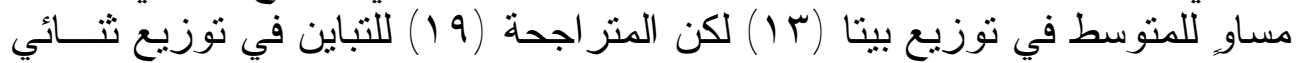

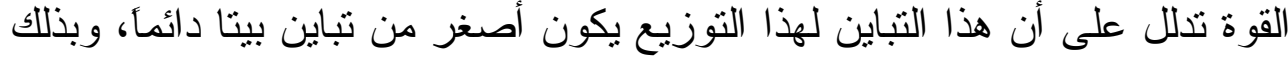

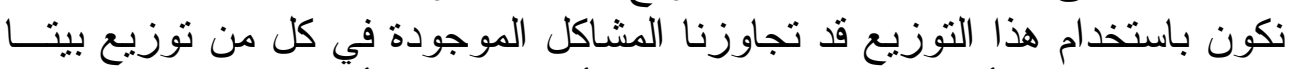

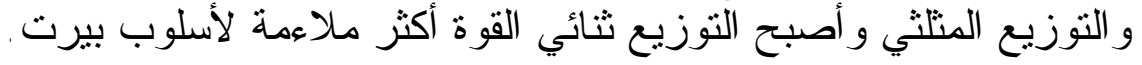

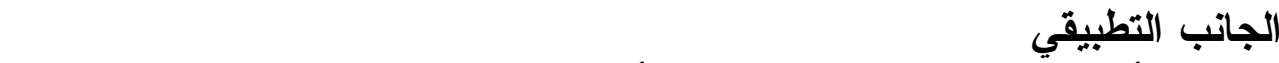

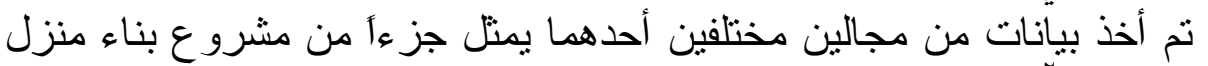

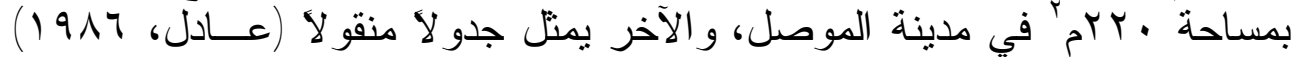

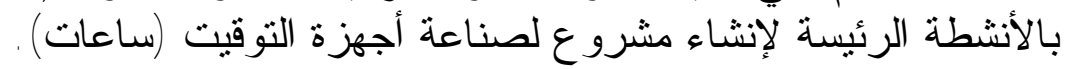

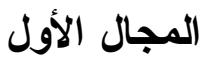

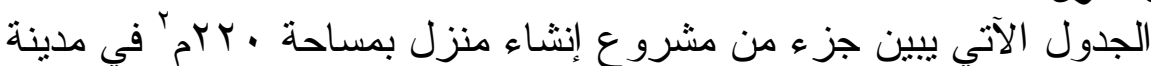
الموصل، وقد نم أَخذ أوقات ستة أنشطة (باليوم) من المشروع. 


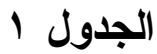

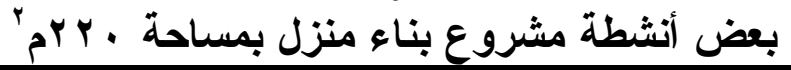

\begin{tabular}{|c|c|c|c|}
\hline الوقت التشاؤمي & الوقت الأكثر احتمالا & التفاؤلي & النشاط \\
\hline$\overline{3}$ & $\overline{2}$ & 1 & شر اء مو اد للمجاري \\
\hline 3 & $\overline{2}$ & 1 & شر اء مو اد كهربائية \\
\hline 5 & $\overline{4}$ & $\overline{3}$ & تركيب مجاري \\
\hline$\overline{9}$ & $\overline{5}$ & 4 & تركيب أسلاك الكهرباء \\
\hline 5 & $\overline{4}$ & $\overline{3}$ & ربط متعلقات المجاري \\
\hline 4 & $\overline{3}$ & 2 & ربط الدصابيح الكهربائية \\
\hline
\end{tabular}

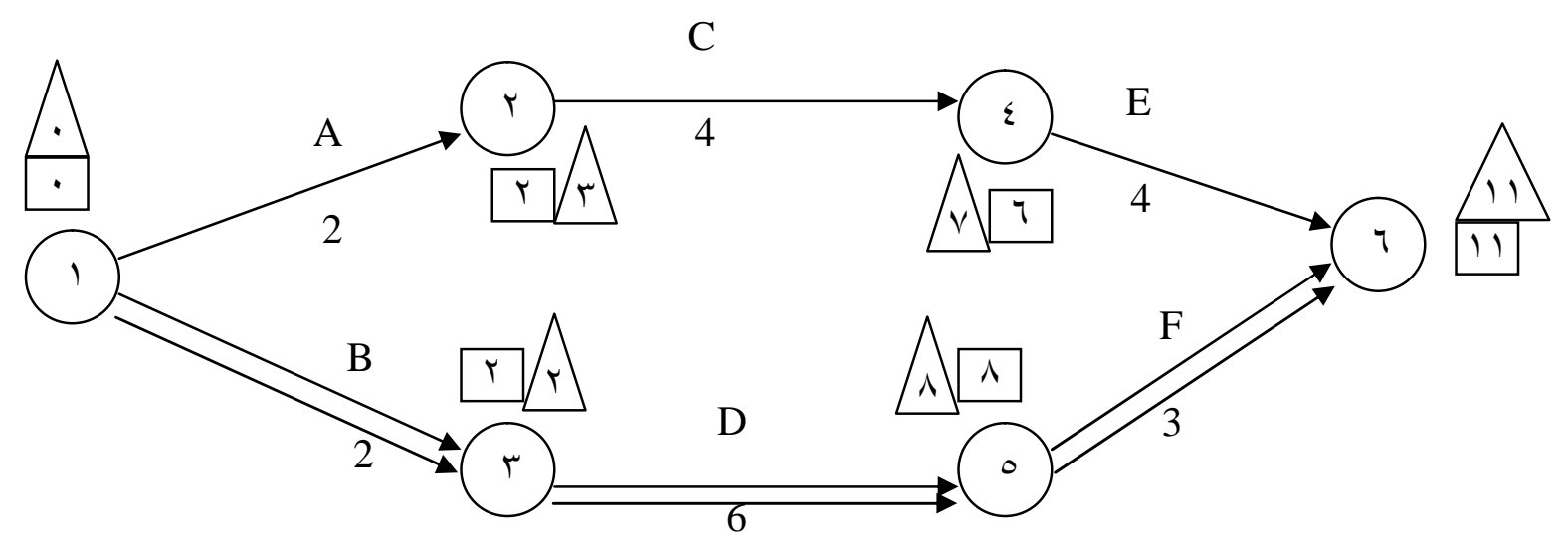

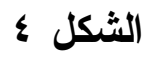

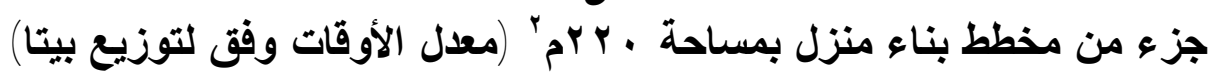

$$
\text { وحيث أن }
$$

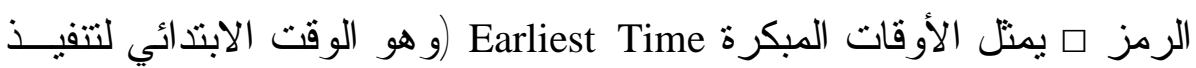

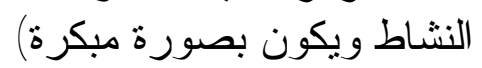

Latest Event Time الرمز ه يمتل الأوقات الأخيرة لمبانشرة الفعاليات

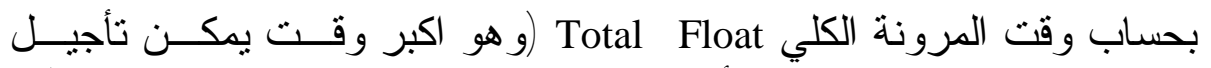
المبانشرة في تتفيذ نشاط ما من دون أن يؤثر ذلك في وقت إكمال المشروع الكلي). $\mathrm{TFij}=\mathrm{LCj}-\mathrm{Esi}-\mathrm{tij}$ 


\section{[إدريس [900}

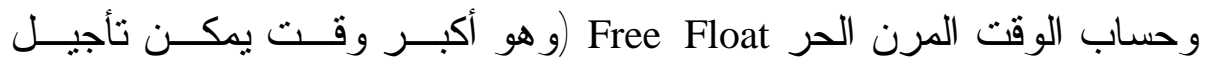

المباثرة بتنفيذ نشاط ما إذا ابتدأت الأنشطة الباقية كافة في الأوقات المكتوبة لها. $F F i j=E S j-E s i-t i j$

تم تكوين الثبكة وحساب الاحتمالات الممكنة باعتماد على القول بأن الأنشطة

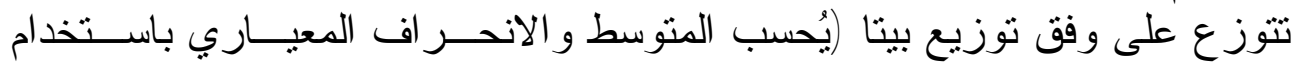

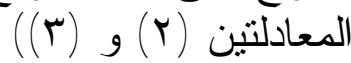

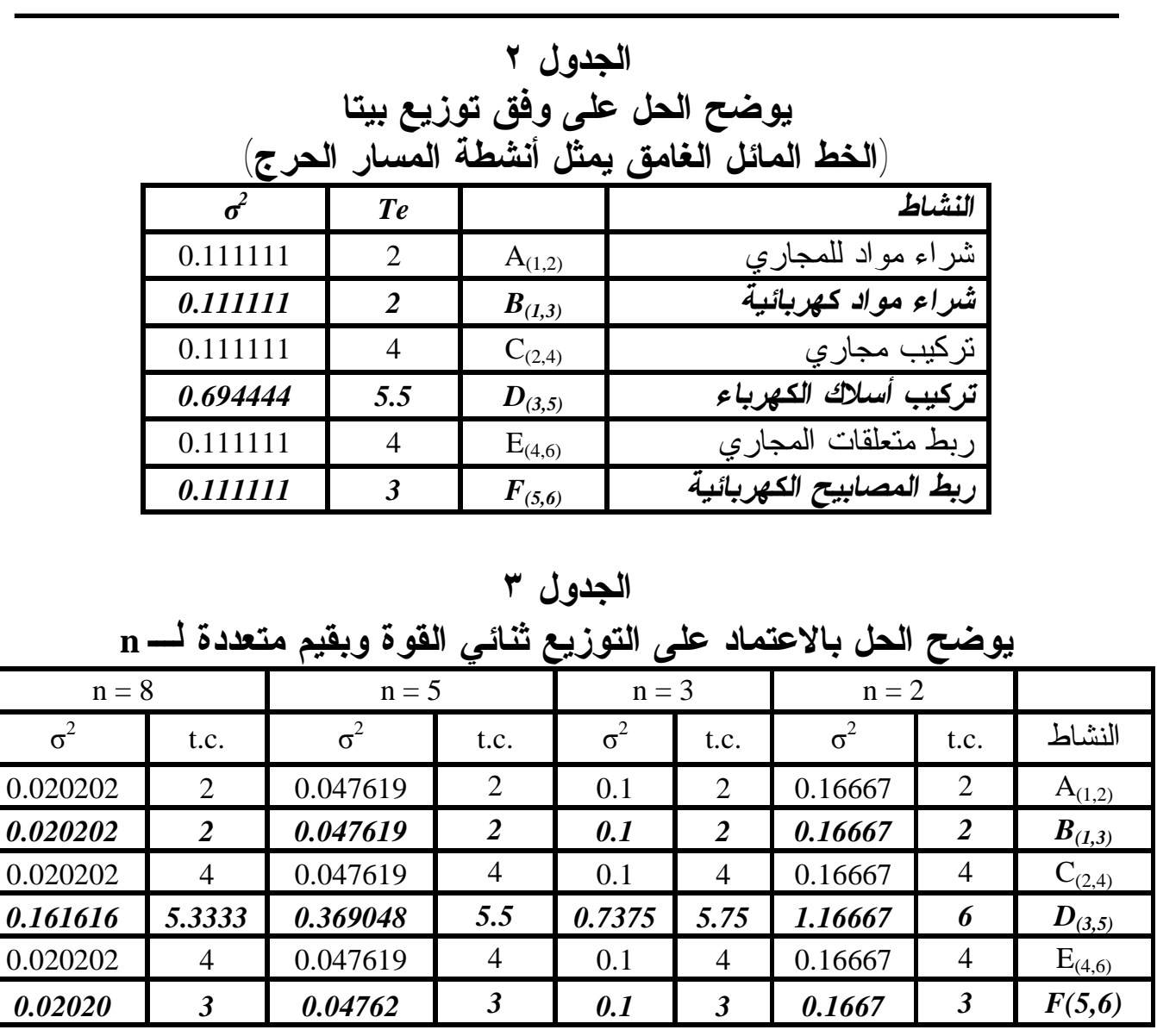

أما بالنسبة لمجموع التباينات للمسار الحرج (B - D - F) فهي علـى وفـق

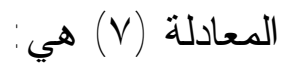




\begin{tabular}{|c|c|c|c|c|}
\hline \multicolumn{5}{|c|}{ الجدول \& (*) } \\
\hline$\Lambda$ & 0 & $r$ & $r$ & $\bar{N}$ \\
\hline 0.20202 & 0.9375 & 1.5 & 0.464286 & $\Sigma_{(\mathrm{c} . \mathrm{p})}^{2}$ \\
\hline
\end{tabular}

(نلاحظ أن التباينات للتوزيع ثنائي القوة للأنشطة أقل من تباين توزيع بيتـا

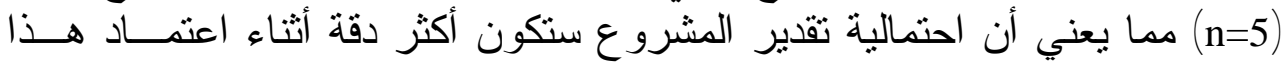

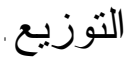

يوضح الجدول الآتي الأنشطة الرئيسة لمشروع إنتاج أجهزة نوقيت (ساعات) | المجال الثاني

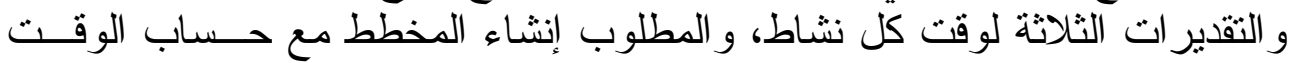
المتوقع لكل نشاط و التباين الخاص بله به وله

\section{الجدول}

أنشطة المشروع مع محتوياتها

\begin{tabular}{|c|c|c|c|c|c|}
\hline المتشنائم b & m المعتدل m & a المتفائل a & النشاط السابق & وصف النشاط & النشاط \\
\hline 0 & $\varepsilon$ & $r$ & $\cdots$ & التخطيط المبدئي & A \\
\hline$\Lambda$ & 7 & $\varepsilon$ & $\mathrm{A}$ & در اسة السوق & $\mathrm{B}$ \\
\hline 9 & $\mathrm{~V}$ & 0 & $\mathrm{~B}$ & التصميم المبدئي للمنتج & $\mathrm{C}$ \\
\hline $1 \varepsilon$ & 9 & $\varepsilon$ & $\mathrm{C}$ & تصميم الجزء الميكانيكي & $\mathrm{D}$ \\
\hline r & r & 1 & $\mathrm{C}$ & تصميم الجزء الكهربائي & $\mathrm{E}$ \\
\hline 7 & $\varepsilon$ & $r$ & $\mathrm{D}$ & تصميم نموذج الجزء الميكانيكي & $\mathrm{F}$ \\
\hline$r$ & r & $\bar{T}$ & $\mathrm{E}$ & تصميم نموذج الجزء الكهربائي & $\bar{G}$ \\
\hline 1 & 1 & 1 & $\mathrm{~F}, \mathrm{G}$ & تجميع الجز عين معاً & $\mathrm{H}$ \\
\hline$\Lambda$ & 0 & r & $\mathrm{H}$ & الاختيار المبدئي للأنموذج & I \\
\hline $1 \varepsilon$ & $1 \cdot$ & 7 & $\mathrm{I}$ & تجهيز الرسومات التفصيلية & $\bar{J}$ \\
\hline $\mathrm{V}$ & 0 & $r$ & $\mathbf{J}$ & اختيار المكائن و الآلات & $\mathrm{K}$ \\
\hline 9. & 7. & r. & $\mathrm{K}$ & طلب الأدو ات و المعدات & $\mathrm{L}$ \\
\hline $1 \cdots$ & 9. & $\Lambda$. & $\mathrm{J}$ & بناء المصنع & $\mathrm{M}$ \\
\hline$r \cdot$ & $1 \varepsilon$ & $1 \varepsilon$ & $\mathrm{L}, \mathrm{M}$ & تزكيب المعدات و المكائن في المصنع & $\mathrm{N}$ \\
\hline
\end{tabular}

(") القيم في الجدول ناتج جمع أنشطة المسار الحرج فقط (الأنشطة ذوات اللون الغامق). 
[إدريس [Y [Y ]

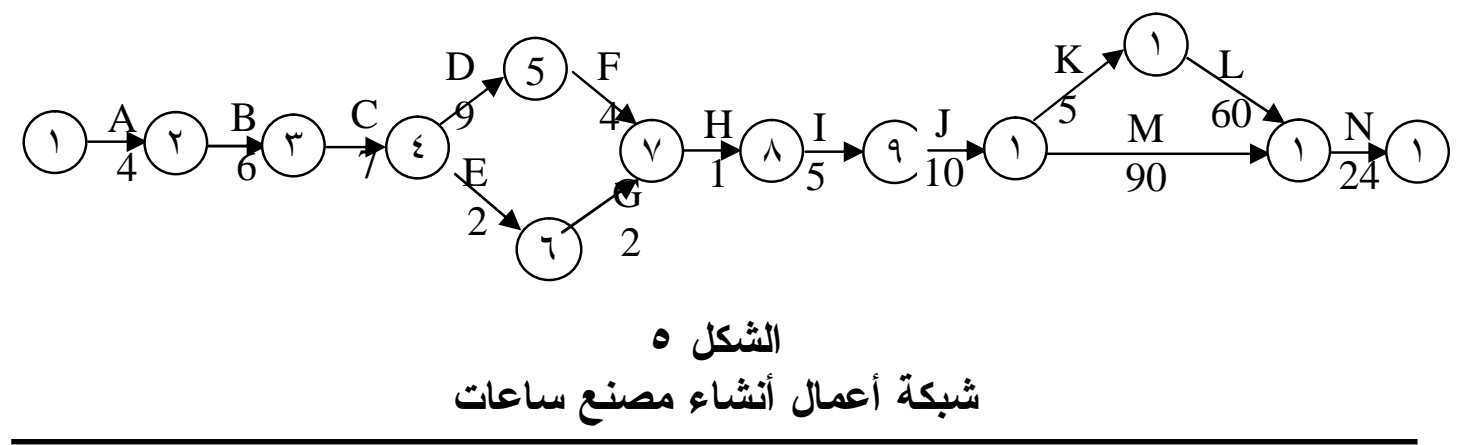

\section{الجدول}

حساب المعدل والتباين للأشطة بالاعتماد على توزيع بيتا

\begin{tabular}{|c|c|c|c|c|c|c|c|}
\hline التباين & المعدل & b المنشائم b & المعتدل m & a المتفائل a & النشاط السابق & وصف النشاط & النشاط \\
\hline 0.1111 & $\varepsilon$ & 0 & $\varepsilon$ & $\mu$ & $\cdots$ & التخطيط المبلئسي & $\bar{A}$ \\
\hline 0.4444 & 7 & $\Lambda$ & 7 & $\varepsilon$ & $A$ & دراسة السوق & $B$ \\
\hline 0.4444 & $V$ & 9 & $V$ & 0 & $B$ & التصميم المبلئسي للمنتج & $C$ \\
\hline 2.7778 & 9 & $1 \leq$ & 9 & $\varepsilon$ & $C$ & تصميم الجزء الميكاتيكي & $D$ \\
\hline 0.1111 & r & $r$ & $\bar{r}$ & $\overline{1}$ & $\bar{C}$ & تصميم الجزء الكهربائي & $\bar{E}$ \\
\hline 0.4444 & $\varepsilon$ & 7 & $\varepsilon$ & $r$ & $\bar{D}$ & تصميم أ نموذج الجزء الميكاتيكي & $\bar{F}$ \\
\hline 0.1111 & r & $r$ & r & 1 & E & تصميم نموذج الجزء الكهربائي & G \\
\hline 0 & 1 & 1 & 1 & 1 & $F, G$ & تجميع الجزعين معاً & $\boldsymbol{H}$ \\
\hline 1 & 0 & $\Lambda$ & 0 & $r$ & $H$ & الاختيار المبائسي للنموذج & $I$ \\
\hline 1.7778 & 1. & $1 \leq$ & 1. & 7 & $\bar{I}$ & تجهيز الرسومات التفصيلية & $J$ \\
\hline 0.4444 & 0 & $\mathrm{~V}$ & 0 & $r$ & $\mathrm{~J}$ & اختبار المكائن و الآلات & $\mathrm{K}$ \\
\hline 100 & 7. & 9. & 7. & r. & $\mathrm{K}$ & طلب الأدو ات و المعدات & $\mathrm{L}$ \\
\hline 11.111 & 9. & $1 \ldots$ & 9. & $\Lambda$. & $J$ & بناء المصنع & $M$ \\
\hline 7.1111 & $r \varepsilon$ & $r$. & $1 \leq$ & $1 \leq$ & $L, M$ & تركيب المعدات والمكائن في المصنع & $N$ \\
\hline
\end{tabular}




\section{الجدول}

حساب المعدل و التباين للأشطة بالاعتماد على توزيع ثنائي القوة

\begin{tabular}{|c|c|c|c|c|c|c|c|c|c|}
\hline \multicolumn{2}{|c|}{$\mathrm{n}=8$} & \multicolumn{2}{|c|}{$n=5$} & \multicolumn{2}{|c|}{$\mathrm{n}=3$} & \multicolumn{2}{|c|}{$\mathrm{n}=2$} & \multirow[b]{2}{*}{ النشاط السابق } & \multirow[b]{2}{*}{ النشاط } \\
\hline 0.020202 & 4 & 0.047619 & 4 & 0.1 & 4 & 0.1667 & 4 & & \\
\hline 0.080808 & 6 & 0.190476 & 6 & 0.4 & 6 & 0.6667 & 6 & $\cdots$ & A \\
\hline 0.080808 & 7 & 0.190476 & 7 & 0.4 & 7 & 0.6667 & 7 & $\bar{A}$ & $\mathrm{~B}$ \\
\hline 0.505051 & 9 & 1.190476 & 9 & 2.5 & 9 & 4.1667 & 9 & B & $\mathrm{C}$ \\
\hline 0.020202 & 2 & 0.047619 & 2 & 0.1 & 2 & 0.1667 & 2 & $\mathrm{C}$ & $\mathrm{D}$ \\
\hline 0.080808 & 4 & 0.190476 & 4 & 0.4 & 4 & 0.6667 & 4 & $\mathrm{C}$ & E \\
\hline 0.020202 & 2 & 0.047619 & 2 & 0.1 & 2 & 0.1667 & 2 & $\mathrm{D}$ & $F$ \\
\hline 0 & 1 & 0 & 1 & 0 & 1 & 0 & 1 & $\mathrm{E}$ & G \\
\hline 0.181818 & 5 & 0.428571 & 5 & 0.9 & 5 & 1.5 & 5 & $\mathrm{~F}, \mathrm{G}$ & $\mathrm{H}$ \\
\hline 0.323232 & 10 & 0.761905 & 10 & 1.6 & 10 & 2.6667 & 10 & $\mathrm{H}$ & $\mathrm{I}$ \\
\hline 0.080808 & 5 & 0.190476 & 5 & 0.4 & 5 & 0.6667 & 5 & I & $\mathrm{J}$ \\
\hline 18.18182 & 60 & 42.85714 & 60 & 90 & 60 & 150 & 60 & $\mathrm{~J}$ & K \\
\hline 2.020202 & 90 & 4.761905 & 90 & 10 & 90 & 16.667 & 90 & $\mathrm{~K}$ & $\mathrm{~L}$ \\
\hline 1.434343 & 24.33 & 3.333333 & 24 & 6.85 & 23.5 & 11.167 & 23 & $\mathrm{~J}$ & $\mathrm{M}$ \\
\hline 0.020202 & 4 & 0.047619 & 4 & 0.1 & 4 & 0.1667 & 4 & $\mathrm{~L}, \mathrm{M}$ & $\mathrm{N}$ \\
\hline
\end{tabular}

\section{V الجدول}

قيم التباين المقابلة لقيم n في توزيع ثنائي القوة

\begin{tabular}{|c|c|c|c|c|}
\hline$\wedge$ & 0 & $r$ & $r$ & $\mathrm{n}$ \\
\hline 0.323232 & 0.761905 & 1.6 & 2.666667 & $\sigma_{(\mathrm{c}, \mathrm{p})}^{2}$ \\
\hline
\end{tabular}

نلاحظ من الجدول 7 أن القيمة الدتوقعة لإنجاز المشروع قد تزداد أو تقل

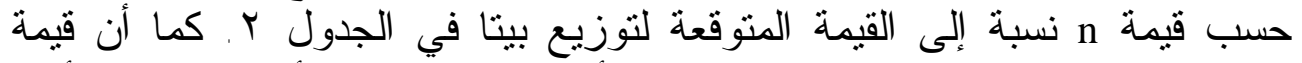

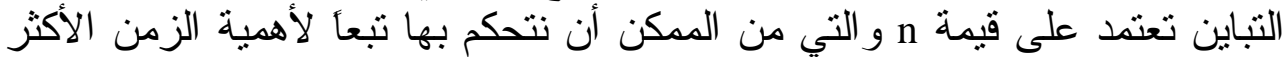

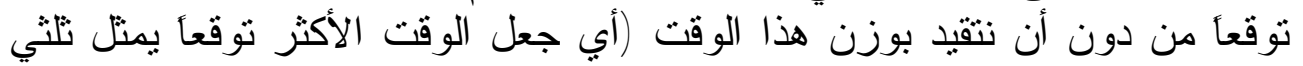

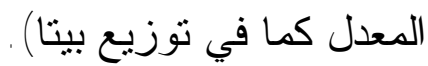

\section{| - الاستنتاجات}

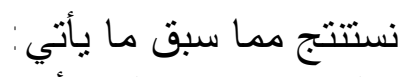

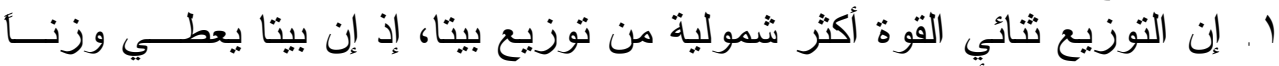

$$
\text { محددأ للوقت الأكثر احتمالأ. }
$$

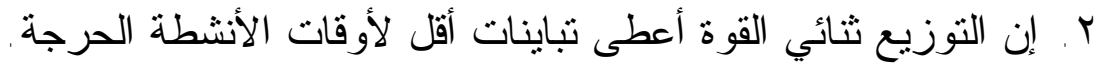

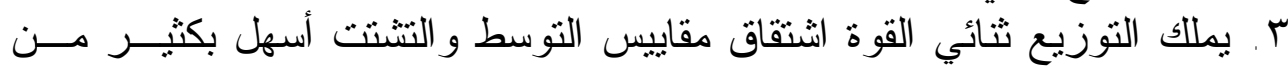

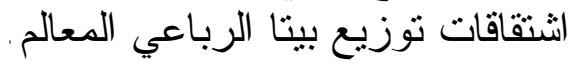


إدريس

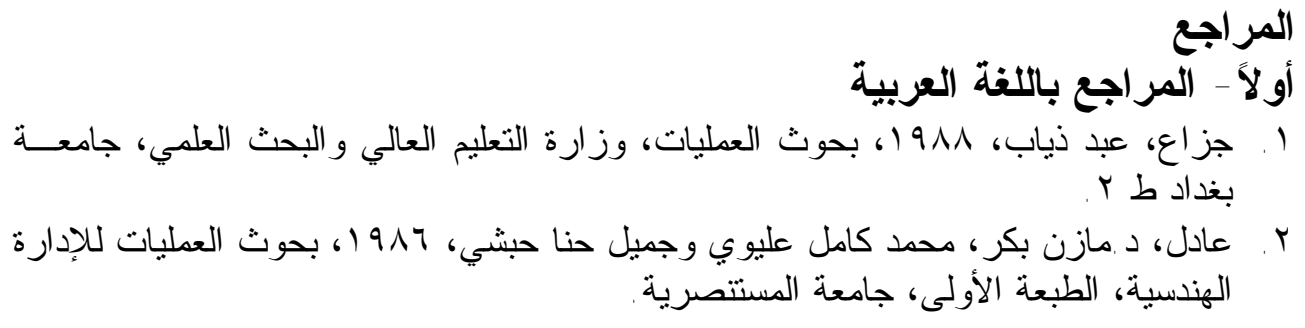

\section{ثانياً - المر اجع باللغة الأجنبية}

1. Bradley, A. Hanson 1991 "Method of Moments Estimates for the Four-Parameter Beta Compound Binomial Model and the Calculation of Classification Consistency Indexes".ACT Reseaech Report Series.

2. Duffey, M.R. \& Van Dorp, J. R. 2000 " Risk Analysis for Large Engineering Projects: Modeling Cost Uncertainty for Ship Production Activities" Journal of Engineering Valuation and Cost Analysis, Vol. 2.

3. Van Dorp, J.R., \& Kotz, S. 2002, "the standard two - sided power distribution and its properties with applications in Financial engineering". The American Statistician, 56, 90 $-99$.

4. Van Dorp, J.R., \& Kotz, S. 2002, "A Versatile Bivariate Distribution on a Bounded Domain Another Look at the product Moment Correlation". Journal of Applied Statistics, Vol. 29, No. 8. 